\title{
Oxalobacter formigenes-associated host features and microbial community structures examined using the American Gut Project
}

Menghan Liu' ${ }^{1,3,4}$, Hyunwook Koh ${ }^{2 \dagger}$, Zachary D. Kurtz ${ }^{3,4+}$, Thomas Battagliaa ${ }^{3,4}$, Amanda PeBenito ${ }^{3,4}$, Huilin Li ${ }^{2}$, Lama Nazzal $\left.\right|^{3,4^{*}}$ and Martin J. Blaser ${ }^{3,4,5^{*}}$

\begin{abstract}
Background: Increasing evidence shows the importance of the commensal microbe Oxalobacter formigenes in regulating host oxalate homeostasis, with effects against calcium oxalate kidney stone formation, and other oxalateassociated pathological conditions. However, limited understanding of $O$. formigenes in humans poses difficulties for designing targeted experiments to assess its definitive effects and sustainable interventions in clinical settings. We exploited the large-scale dataset from the American Gut Project (AGP) to study O. formigenes colonization in the human gastrointestinal (Gl) tract and to explore $O$. formigenes-associated ecology and the underlying host-microbe relationships.
\end{abstract}

Results: In >8000 AGP samples, we detected two dominant, co-colonizing O. formigenes operational taxonomic units (OTUs) in fecal specimens. Multivariate analysis suggested that $O$. formigenes abundance was associated with particular host demographic and clinical features, including age, sex, race, geographical location, BMI, and antibiotic history. Furthermore, we found that $O$. formigenes presence was an indicator of altered host gut microbiota structure, including higher community diversity, global network connectivity, and stronger resilience to simulated disturbances.

Conclusions: Through this study, we identified O. formigenes colonizing patterns in the human Gl tract, potential underlying host-microbe relationships, and associated microbial community structures. These insights suggest hypotheses to be tested in future experiments. Additionally, we proposed a systematic framework to study any bacterial taxa of interest to computational biologists, using large-scale public data to yield novel biological insights.

Keywords: Ecology, Gut microbiota, Microbial network, Host-microbe interaction, Kidney stones, Systems biology, Public data mining

\section{Background}

Oxalate is both a dietary constituent [1] and a product of endogenous human metabolism [2,3]. Excessive oxalate accumulation can promote pathological conditions, including kidney stones [4-6], joint effusions, arthralgias $[7,8]$, and breast cancer [9]. However, unable to catabolize oxalate, humans rely on oxalate degradation

\footnotetext{
* Correspondence: Lama.Nazzal@nyumc.org; Martin.Blaser@nyumc.org ${ }^{\dagger}$ Equal contributors

${ }^{3}$ Department of Microbiology, New York University School of Medicine, New York, NY 10016, USA

Full list of author information is available at the end of the article
}

performed by commensal bacteria and intestinal and urinary excretion to decrease the circulating oxalate levels [1].

Oxalobacter formigenes degrades oxalate as its sole energy and carbon source [10] within the gastrointestinal (GI) tract of its hosts, in contrast to other known oxalate-degrading bacteria, nearly all of which only metabolize oxalate using detoxification pathways under specific conditions $[11,12]$. A second physiologic role of $O$. formigenes in host homeostasis is in stimulating 
oxalate transport through the gut epithelium, promoting its release into the GI tract lumen [13].

Although the potential of $O$. formigenes to remove excessive oxalate from the host has been long recognized [14-19], re-introduction of the organism to humans have not yielded definitive results $[15,17,20,21]$; this may reflect the insufficiency of in vivo models, small study sizes, failure to select proper $O$. formigenes strains, and/or the lack of sustained colonization.

In the present study, we aimed to examine $O$. formigenes colonization patterns in humans and to explore the underlying ecological relationships using the American Gut Project (AGP). The AGP has surveyed the intestinal microbiome in more than 8000 people using standard pipelines, and with detailed host metadata, which permits studies of $O$. formigenes-centered ecology. Our study illustrates a systematic framework to examine key bacteria present in large public datasets to ascertain their biological relationships with their hosts.

\section{Results}

\section{Detection of 0 . formigenes OTUs in AGP samples}

In total, 9746 and 9550 AGP samples were processed using QIIME's closed- and open-reference operational taxonomic unit (OTU)-picking methods [10, 22, 23] (Additional file 1: Figure S1) yielding a total of 3 and 260 OTUs, respectively, that were classified as $O$. formigenes by the closed- and open-reference OTU-picking methods
(Additional file 1: Figure S1). Samples with $\geq 1000$ seqs/ sample were used for the initial examination of $O$. formigenes prevalence and abundance (Table 1). The three OTUs that were detected by both methods were Greengenes [24] OTUs 7366, 360508, and 7369 (Table 1); the other 257 OTUs were detected only by the open-reference OTU-picking method at low abundance, each accounting for $<1 \%$ of the total $O$. formigenes-associated reads (Table 1). As such, results of the closed-reference OTU-picking method were used for downstream analyses.

The most dominant OTU 7366 was detected in $27.6 \%$ of all samples, accounting for $96.4 \%$ of the O. formigenes-associated sequencing reads (Table 1). OTU 360508 was detected in $7 \%$ of the samples (Table 1), accounting for $3.5 \%$ of the total $O$. formigenes reads. OTU 7369 was detected in $<1 \%$ of the subjects (Table 1 ).

O. formigenes was chiefly found in fecal samples (Table 2), consistent with prior findings on a smaller scale $[10,15]$; as such, we focused only on fecal samples in subsequent analyses. In fecal samples in which OTU 7366 was detected, its geometric mean relative abundance was $2.9 \times 10^{-4}$ and ranged from $10^{-6}$ to $10^{-3}$. For OTUs 360508 and 7369 , the mean relative abundances were $\sim 10$-fold lower in the samples in which they were detected (Fig. 1a).

\section{Classification of 0 . formigenes OTUs}

O. formigenes strains studied to date have been divided into two subgroups based on biological heterogeneity,

Table 1 Abundance and prevalence of O. formigenes-related OTUs in the American Gut Project

\begin{tabular}{|c|c|c|c|c|c|c|}
\hline \multirow[t]{3}{*}{ OTU ID } & \multicolumn{3}{|c|}{ Closed-reference OTU picking } & \multicolumn{3}{|c|}{ Open-reference OTU picking ${ }^{a}$} \\
\hline & \multirow{2}{*}{$\begin{array}{l}\text { Counts\%/ } \\
\text { cumulative } \\
\text { counts\% }\end{array}$} & \multicolumn{2}{|c|}{$\%$ of colonization } & \multirow{2}{*}{$\begin{array}{l}\text { Counts\%/ } \\
\text { cumulative } \\
\text { counts\% }\end{array}$} & \multicolumn{2}{|c|}{$\%$ of colonization } \\
\hline & & 8610 samples & 7293 subjects & & 8441 samples & 7115 subjects \\
\hline 7366 & $96.43 / 96.43$ & 27.55 & 30.25 & $90.90 / 90.90$ & 27.26 & 30.01 \\
\hline 360508 & $3.48 / 99.91$ & 7.03 & 7.84 & 2.04/92.93 & 5.01 & 5.68 \\
\hline New.CleanUp.ReferenceOTU314026 & - & - & - & $0.77 / 93.70$ & 2.11 & 2.42 \\
\hline 2641606 & - & - & - & $0.76 / 94.45$ & 1.39 & 1.57 \\
\hline New.ReferenceOTU11344 & - & - & - & $0.68 / 95.13$ & 1.91 & 2.26 \\
\hline 3488180 & - & - & - & $0.55 / 95.68$ & 1.77 & 2.05 \\
\hline New.CleanUp.ReferenceOTU2018399 & - & - & - & $0.50 / 96.18$ & 1.45 & 1.64 \\
\hline New.CleanUp.ReferenceOTU6125098 & - & - & - & $0.23 / 96.41$ & 0.65 & 0.77 \\
\hline 4474081 & - & - & - & $0.21 / 96.62$ & 0.57 & 0.62 \\
\hline New.CleanUp.ReferenceOTU9098367 & - & - & - & $0.20 / 96.81$ & 0.60 & 0.69 \\
\hline New.CleanUp.ReferenceOTU781422 & - & - & - & $0.19 / 97.00$ & 0.58 & 0.69 \\
\hline 191145 & - & - & - & $0.15 / 97.15$ & 0.53 & 0.62 \\
\hline New.CleanUp.ReferenceOTU5660629 & - & - & - & $0.10 / 97.25$ & 0.31 & 0.37 \\
\hline 7369 & $0.09 / 100.00$ & 0.34 & 0.38 & $0.10 / 97.35$ & 0.39 & 0.45 \\
\hline Control: B. fragilis OTU 4479397 & - & 30.00 & 35.00 & - & 45.00 & 49.00 \\
\hline
\end{tabular}

OTUs were ordered from the highest to lowest sequencing read number

${ }^{a} \mathrm{~A}$ total of $260 \mathrm{O}$. formigenes-related OTUs were detected with open-reference OTU picking, and only the 14 most abundant OTUs, representing $97.35 \%$ of all counts, are shown in this table 
Table 2 Prevalence of three O. formigenes OTUs in 8610 samples (only samples with >1000 reads are included) by body site

\begin{tabular}{lllll}
\hline OTU & \multicolumn{3}{l}{ Prevalence $(\%)$ of $O$. formigenes-colonized samples } & \\
\cline { 2 - 5 } & Feces $(n=7420)$ & Skin $(n=322)$ & Tongue $(n=448)$ & Other or unknown sites $(n=310)$ \\
\hline 7366 & 31.6 & 3.1 & 0.9 & 10.0 \\
360508 & 8.1 & 0.3 & 0.2 & 1.6 \\
7369 & 0.4 & 0.2 & 0 & 0 \\
\hline
\end{tabular}

including cellular fatty acid content $[15,25]$ and length variation of key genes (frc and oxc) [26]. Based on the full $16 \mathrm{~S}$ rRNA sequences, the 13 group I and group II $O$. formigenes strains also cluster into distinct clades by a deep branching of the phylogenetic tree (Additional file 2: Figure S2A). The AGP O. formigenes OTUs differ in their $16 \mathrm{~S} \mathrm{~V} 4$ sequence similarity to the 13 reference O. formigenes strains (Fig. 1b). The V4 region of OTU 7366 is $100 \%$ identical to that of O. formigenes strain BLISS (Additional file 2: Figure S2B), initially isolated from human feces in 1996 [27, 28]. Strain BLISS is located on a separate branch of the phylogenetic tree but shares a common root with all group I O. formigenes strains (Additional file 2: Figure S2A). OTUs 360508 and 7366 are most similar to group II $O$. formigenes strains HOxBLS and

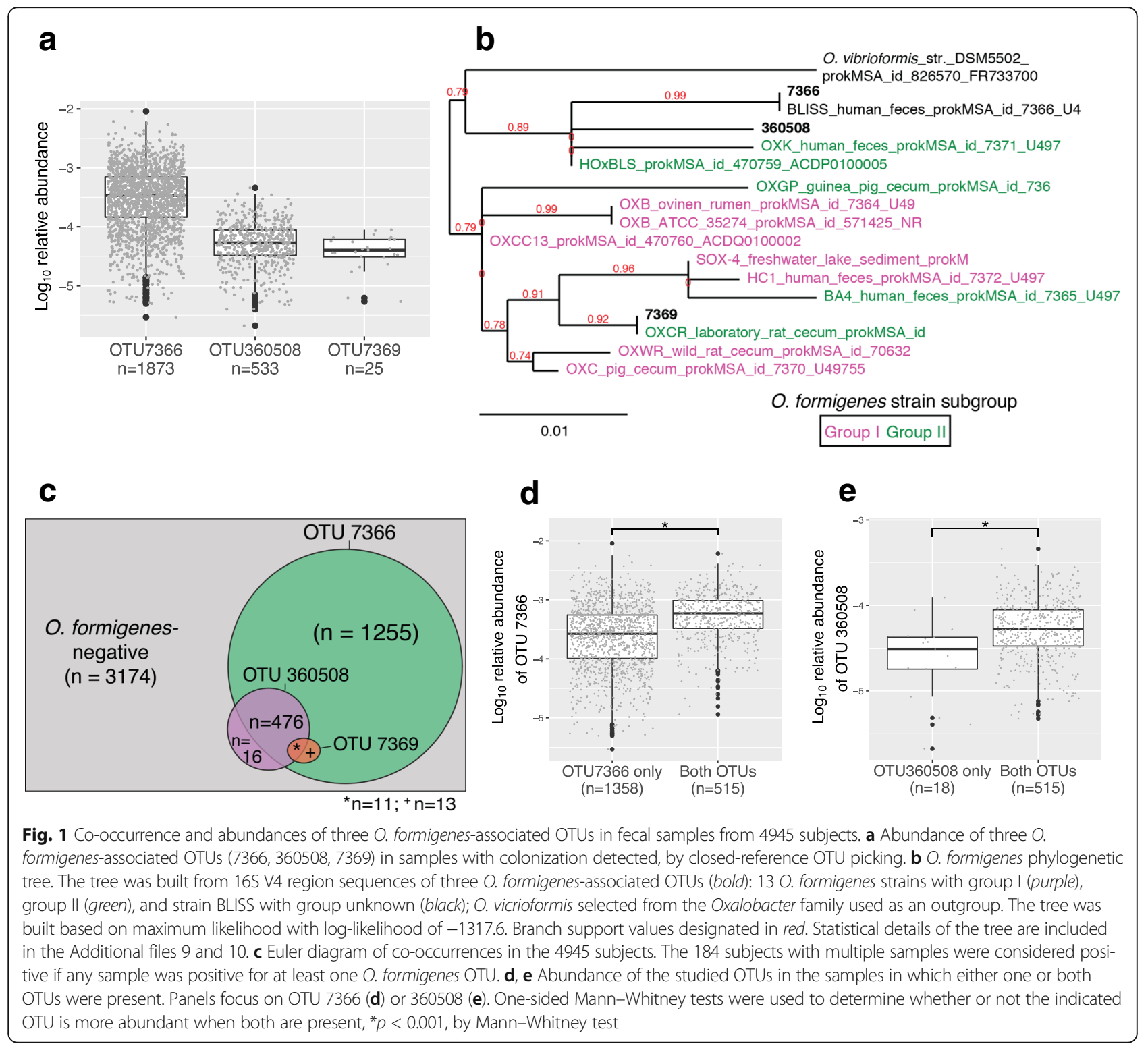


OXCR, at 98.8 and $100 \%$ identity, respectively (Fig. 1b, Additional file 2: Figure S2B).

\section{Selection of samples for downstream analysis}

More O. formigenes OTUs were detected as sequence depth became higher (Additional file 3: Figure S3A). To alleviate potential undersampling, we raised inclusion criteria from $\geq 1000$ seqs/sample (Additional file 3: Figure S3A) to $\geq 10,000$ (Additional file 3: Figure S3B), which included 5336 fecal specimens from 4945 subjects. Among those, we focused on the 4945 AGP fecal specimens, with the subjects who provided multiple specimens ( $n=184$; Additional file 4: Figure S4) represented only by the first specimen provided.

\section{Co-detection of $O$. formigenes}

Since our preliminary analyses suggested that the observed $O$. formigenes OTUs may differ from known $O$. formigenes strains (Fig. 1b), we reasoned that interactions between those OTUs should provide broad insight into the intraspecies $O$. formigenes dynamics. Frequent co-colonization of the two dominant OTUs was observed (Fig. 1c) $\left(p<10^{-27}\right.$, significance of overlap test performed via [29]). In $96.8 \%$ of the samples in which OTU 360508 was detected, OTU 7366 was co-detected. Similarly, OTU 7369 completely overlapped with the samples in which OTUs 7366 and 360508 were detected (Fig. 1c). The relative abundances of both OTUs 7366 (Fig. 1d, $p<0.001$ ) and 360508 (Fig. 1e, $p<0.001$ ) were significantly elevated when co-detected.

\section{Longitudinal colonization of $O$. formigenes}

To investigate $O$. formigenes colonization over time, the 184 subjects who provided multiple fecal samples (Additional file 4: Figure S4) were divided into three groups depending on whether $O$. formigenes was detected in the following: (i) none of the samples ( $n=100$ subjects), (ii) at least one sample ( $n=44$ subjects), or (iii) all the samples ( $n=40$ subjects) provided (Fig. 2a). OTU 7366 was significantly more abundant in samples with detection from group III than from group II (Fig. 2b, left). A parallel trend was noted for OTU 360508 (Fig. 2b, middle) and for the sum of both OTUs (Fig. 2b, right). These observations were likely not due to sequencing depth bias, since total sequences/sample did not differ between samples with detection from groups II and III ( $p$ value $=0.96$, Mann-Whitney test).

\section{Host features associated with detection of $O$. formigenes colonization}

Previous studies [14, 30-32] examining the association of $O$. formigenes colonization with age, sex, and antibiotic exposure history were conducted in small populations. The AGP, with large sample size and detailed metadata available, is a robust data source to explore the underlying $O$. formigenes-host relationships. Based on the prior literature, data availability, and biological relevance, we focused on 14 candidate covariates (Table 3) describing host demographic and clinical features to predict $O$. formigenes abundance.

Univariate analyses between covariates and $O$. formigenes abundance were performed for each covariate independently using the 4945 samples (Table 3). To avoid the confounding effects from highly correlated covariates and to minimize false discovery, we further performed multivariate analysis using a multiple zero-inflated negative binomial (ZINB) model [33]. The rationales for model selection and strategies were described in detail in the "Methods" section. The fitted ZINB model consists of two different components, the logistic regression for modeling excessive zero abundances and the negative binomial regression for modeling the remaining count values. Here, we refer to the population for excessive zero abundances as the population from which O. formigenes is not detected and the other population for the remaining abundances as the population in which it is detected [34]. Based on the fitted ZINB model, we found that age, sex, race, BMI, alcohol drinking frequency, antibiotic use history, country of residence, and level of education are significantly associated with the probability of $O$. formigenes detection by the logistic regression component (Table 4) and BMI and thyroid status are significantly associated with $O$. formigenes detection by the negative regression component (Table 4) [34].

To estimate the overall effect direction and magnitude, we calculated the overall fitted mean proportions (\%) (see the "Methods" section) as measurements of O. formigenes relative abundance for the nine covariates that were significant in either the logistic or negative binomial regression component (Fig. 3, Table 4) [35]. To summarize the outcomes, adjusted for the other covariates, we estimate that relative abundance of $O$. formigenes is associated with increased age (Fig. 3a), female sex (Fig. 3b), Caucasian ethnicity (compared to Asians, Pacific Islanders, Hispanics, African Americans, or for persons of other ethnicities) (Fig. 3c), non-USA residence (Fig. 3d), normal BMI (compared with underweight, overweight, or obese) (Fig. 3e), absence of antibiotic exposure within a year (Fig. 3f), alcohol consumption (Fig. 3g), higher educational attainment (Fig. $3 \mathrm{~h}$ ), and normal thyroid function (Fig. 3i). In another analysis, we showed relationships of $O$. formigenes presence and the locality of the subject's birth and present residence (Additional file 5: Table S1).

In the AGP, participants could complete the Vioscreen questionnaire [36], a validated dietary instrument calculating dietary intake in the preceding 90 days. We were specifically interested in assessing the relationships 


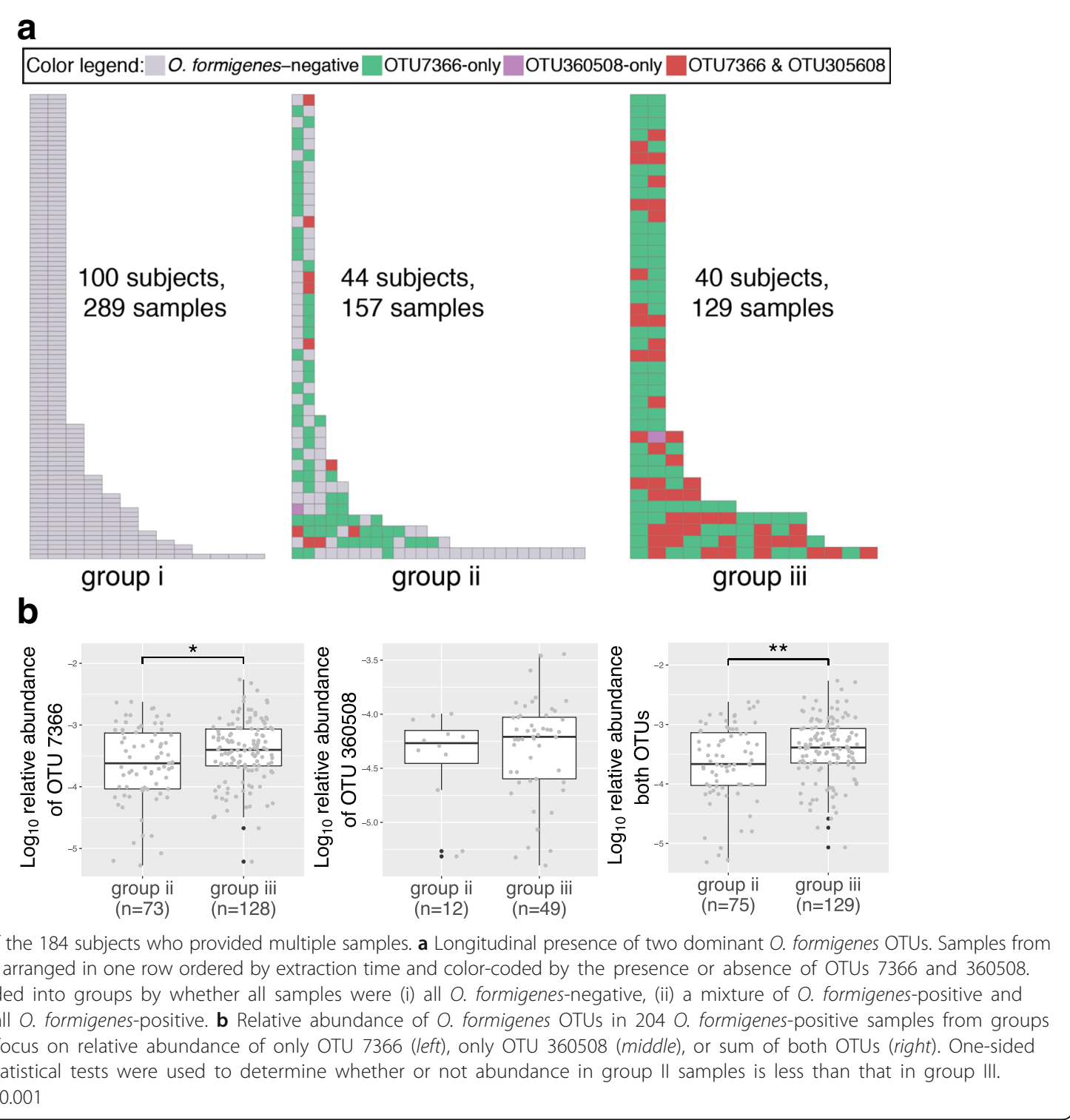

between $O$. formigenes with dietary oxalate and calcium because of the role of oxalate in $O$. formigenes growth and its unavailability when complexed with calcium [1, 5]. Among the 197 participants who provided the questionnaire, $O$. formigenes relative abundance was not significantly associated with dietary oxalate (Additional file 6: Figure S5, left), but was inversely and significantly associated with dietary calcium (Additional file 6: Figure S5, middle; $p=0.028$ by Spearman rank correlation test), and also inversely associated with the ratio of dietary oxalate to calcium (Additional file 6: Figure S5, right; $p=0.002$ ).

\section{Microbial community characteristics in relation to detection of $\boldsymbol{O}$. formigenes}

Among the 4945 subjects, as the number of $O$. formigenes OTUs detected increased, phylogenetic diversity
[37] also increased (Fig. 4a). This relationship was noted when subjects of the USA (Fig. 4b) and UK-Ireland (Fig. 4c) were analyzed independently. The association between increasing $\alpha$-diversity and detection of $O$. formigenes remained consistent when the $\alpha$-diversity was assessed using Chao1 [38], Shannon index [39], or observed number of OTUs, with rarefaction (Additional file 7: Figure S6) to adjust for sequencing depth bias.

To examine microbial community structure $(\beta$-diversity), we built a sample of 824 specimens that met rigorous inclusion criteria (Additional file 5: Table S2). Based on both unweighted UniFrac distances [40] (Fig. 5a) and Bray-Curtis dissimilarities [41] (Additional file 8: Figure S7A) visualized by principal coordinate analysis (PCoA), there was substantial overlap in the samples in which either one or two O. formigenes OTUs were detected. 
Table 3 Descriptive statistics and univariate analyses for 14 candidate covariates for 4945 fecal samples

\begin{tabular}{|c|c|c|c|c|c|}
\hline \multirow{2}{*}{$\begin{array}{l}\text { Covariates } \\
\text { Continuous variable }\end{array}$} & \multicolumn{3}{|c|}{ All samples $(n=4945)$} & \multicolumn{2}{|c|}{$\begin{array}{l}\text { Univariate analysis } \\
p \text { value }^{a}\end{array}$} \\
\hline & Mean \pm SD & $\begin{array}{l}\text { Frequency (\%) } \\
\text { of missing }\end{array}$ & $\begin{array}{l}\text { Mean age of } O \text {. } \\
\text { formigenes-positive/O. } \\
\text { formigenes-negative }\end{array}$ & Logistic & Negative binomial \\
\hline Age & $46.08 \pm 17.28$ & $246(4.97)$ & 48.39:44.78 & $<.001$ & $<.001$ \\
\hline Categorical covariate & Frequency (\%) & $\begin{array}{l}\text { Frequency (\%) } \\
\text { of missing }\end{array}$ & $\begin{array}{l}\text { Frequency (\%) of } O \text {. } \\
\text { formigenes-positive }\end{array}$ & Logistic & Negative binomial \\
\hline Sex & $4722(95.49)$ & $223(4.51)$ & & & \\
\hline Female & 2576 (52.09) & & 897 (34.84) & Reference & \\
\hline Male & $2146(43.40)$ & & 775 (36.11) & 0.791 & 0.224 \\
\hline Race & 4867 (98.42) & 78 (1.58) & & & \\
\hline Caucasian & 4369 (88.35) & & $1602(36.67)$ & Reference & \\
\hline Asian/Pacific Islander & $232(4.69)$ & & $40(17.24)$ & $<.001$ & $<.001$ \\
\hline Hispanic & $86(1.74)$ & & $28(32.56)$ & 0.315 & 0.082 \\
\hline African American & $50(1.01)$ & & $9(18.00)$ & 0.012 & 0.075 \\
\hline Other & $130(2.63)$ & & $41(31.54)$ & 0.178 & 0.674 \\
\hline BMI & $4620(93.43)$ & $325(6.57)$ & & & \\
\hline Underweight & $453(9.16)$ & & $113(24.94)$ & $<.001$ & $<.001$ \\
\hline Normal & $2720(55.01)$ & & $1093(40.18)$ & Reference & \\
\hline Overweight & 985 (19.92) & & 347 (35.23) & 0.003 & $<.001$ \\
\hline Obese & $462(9.34)$ & & $114(24.68)$ & $<.001$ & $<.001$ \\
\hline $\begin{array}{l}\text { Frequency of } \\
\text { alcohol consumption }\end{array}$ & 4855 (98.18) & $90(1.82)$ & & & \\
\hline Never & $1146(23.17)$ & & 317 (27.66) & Reference & \\
\hline $\begin{array}{l}\text { Rarely (a few } \\
\text { times a month) }\end{array}$ & $1228(24.83)$ & & $447(36.40)$ & $<.001$ & 0.038 \\
\hline $\begin{array}{l}\text { Occasionally } \\
\text { (1-2 times/week) }\end{array}$ & $1076(21.76)$ & & $400(37.17)$ & $<.001$ & 0.055 \\
\hline Regularly (3-5 times/week) & $888(17.96)$ & & $343(38.63)$ & $<.001$ & 0.098 \\
\hline Daily & $517(10.46)$ & & $213(41.20)$ & $<.001$ & 0.192 \\
\hline Last exposure to antibiotics & $4822(97.51)$ & $123(2.49)$ & & & \\
\hline >365 days & $3281(66.35)$ & & $1286(39.20)$ & Reference & \\
\hline$<365$ days & $717(14.50)$ & & $223(31.10)$ & $<.001$ & 0.018 \\
\hline$<180$ days & $584(11.81)$ & & $136(23.19)$ & $<.001$ & $<.001$ \\
\hline$<30$ days & $149(3.01)$ & & $44(29.53)$ & 0.070 & 0.317 \\
\hline$<7$ days & $91(1.84)$ & & $23(25.27)$ & 0.002 & 0.369 \\
\hline Presence of appendix & $4784(96.74)$ & $161(3.26)$ & & & \\
\hline No & $4297(86.90)$ & & $1537(35.77)$ & Reference & \\
\hline Yes & $487(9.85)$ & & $160(32.85)$ & 0.170 & 0.347 \\
\hline Country & $4945(100.00)$ & $0(0)$ & & & \\
\hline USA & $3779(76.42)$ & & $1176(31.12)$ & Reference & \\
\hline UK-Ireland & 819 (16.56) & & $411(50.18)$ & $<.001$ & $<.001$ \\
\hline Europe Continental & $111(2.24)$ & & $52(46.85)$ & 0.001 & 0.088 \\
\hline Australia and NZL & $137(2.77)$ & & $71(51.82)$ & $<.001$ & 0.030 \\
\hline Canada & $73(1.48)$ & & $31(42.47)$ & 0.099 & 0.442 \\
\hline Others & $26(0.53)$ & & $13(50.00)$ & 0.099 & 0.656 \\
\hline
\end{tabular}


Table 3 Descriptive statistics and univariate analyses for 14 candidate covariates for 4945 fecal samples (Continued)

\begin{tabular}{|c|c|c|c|c|c|}
\hline Drinking water source & $4821(97.49)$ & $124(2.51)$ & & & \\
\hline City & $2283(46.17)$ & & 855 (37.45) & Reference & \\
\hline Filtered & $1709(34.56)$ & & $587(34.35)$ & 0.169 & 0.389 \\
\hline Bottled & $426(8.61)$ & & $126(29.58)$ & 0.002 & 0.524 \\
\hline Well & $403(8.15)$ & & $136(33.75)$ & 0.366 & 0.928 \\
\hline Level of education & $2791(56.44)$ & $2154(43.56)$ & & & \\
\hline$\leq$ High school & $245(4.95)$ & & $72(29.39)$ & 0.007 & 0.146 \\
\hline College/bachelor's & $1024(20.71)$ & & 405 (39.55) & Reference & \\
\hline Graduate school & $1522(30.78)$ & & $613(40.28)$ & 0.418 & 0.514 \\
\hline Dog & $4834(97.76)$ & $111(2.24)$ & & & \\
\hline Absent & $3362(67.99)$ & & $1219(36.26)$ & Reference & \\
\hline Present & $1472(29.77)$ & & $489(33.22)$ & 0.058 & 0.118 \\
\hline Born by C-section & $4626(93.55)$ & $319(6.45)$ & & & \\
\hline False & $4147(83.86)$ & & $1485(35.81)$ & Reference & \\
\hline True & 479 (9.69) & & $152(31.73)$ & 0.047 & 0.584 \\
\hline Vegetable consumption frequency & $2876(58.16)$ & $2069(41.84)$ & & & \\
\hline$<1$ time/week & $127(2.57)$ & & $33(25.98)$ & 0.017 & 0.034 \\
\hline 1-2 times/week & $288(5.82)$ & & $84(29.17)$ & 0.024 & 0.046 \\
\hline 3-5 times/week & $1025(20.73)$ & & $381(37.17)$ & Reference & \\
\hline Daily & $1436(29.04)$ & & $616(42.90)$ & 0.001 & 0.007 \\
\hline Thyroid disease & $2853(57.69)$ & $2092(42.31)$ & & & \\
\hline No condition & $2490(50.35)$ & & $986(39.60)$ & Reference & \\
\hline Diagnosed & 363 (7.34) & & 120(33.06) & 0.001 & 0.070 \\
\hline
\end{tabular}

However, both significantly differed from the samples in which $O$. formigenes was absent. The mean pairwise intergroup distances between the $O$. formigenes+ and $O$. formigenes- groups were significantly greater than the corresponding intragroup distances within the O. formigenes+ groups (Fig. 5e, Additional file 8: Figure S7E). The same pattern was observed in samples from subjects from the USA (Fig. 5b, f, Additional file 8: Figure S7B, F), or UK-Ireland (Fig. 5c, g, Additional file 8: Figure S7C, G), when analyzed independently. We also compared the true intragroup distances with average intragroup distances from bootstrapping samples but did not see any scalable differences.

\section{Microbial networks in relation to 0 . formigenes colonization}

We next examined the microbial networks using SPIECEASI [42], where the pairwise microbial interactions were estimated via absence of conditional independence, using the stability approach to regularization selection (StARS) method (as implemented in the pulsar package in $\mathrm{R}$ ) for model selection. For StARS, we used the default $\beta=0.05$ as the threshold parameter, which measures the fraction of the network that is $1-\beta$ stable over random subsamples. To predict interactions between $O$. formigenes and other microbial species, we constructed three networks using all 4945 samples, or subsets of the 3935 US or 830 UK samples. Five bacterial species and an Archaeaon (Table 5) were predicted to interact with $O$. formigenes in at least one of the networks. A negative interaction between Ruminococcus gnavus and O. formigenes was observed in all three networks (Table 5).

To understand the observed differences in microbial community structure between $O$. formigenes+ and $O$. formigenes- samples (Fig. 5), we then inferred two separate networks. We first compared the two networks in terms of centrality of nodes, in which higher values indicate that the node is involved in more ecological interactions. Nodes in the O. formigenes+ network had significantly higher degree [43] and betweenness [44] centrality ( $p=0.03$ and 0.02 , onesided Mann-Whitney tests) than in the O. formigenes - network (Fig. 6a), suggesting greater dispersion within the O. formigenes- network. 
Table 4 The outcomes of the logistic and negative binomial components of the fitted ZINB regression model

\begin{tabular}{|c|c|c|c|c|c|c|}
\hline & \multicolumn{3}{|c|}{ Logistic regression component } & \multicolumn{3}{|c|}{ Negative binomial regression component } \\
\hline & Estimate \pm Std. error & $z$ value & $\operatorname{Pr}(>|z|)^{a}$ & Estimate \pm Std. error & $z$ value & $\operatorname{Pr}(>|z|)^{\mathrm{a}}$ \\
\hline Intercept & $10.133 \pm 0.449$ & -22.545 & $<.001$ & $-8.262 \pm 0.240$ & -34.376 & $<.001$ \\
\hline Age & $-0.025 \pm 0.007$ & -3.593 & $<.001$ & $0.004 \pm 0.003$ & 1.108 & 0.268 \\
\hline \multicolumn{7}{|l|}{ Sex } \\
\hline Female & \multicolumn{6}{|c|}{ Reference category } \\
\hline Male & $-0.422 \pm 0.176$ & -2.403 & 0.016 & $-0.122 \pm 0.090$ & -1.348 & 0.178 \\
\hline \multicolumn{7}{|l|}{ Race } \\
\hline Caucasian & \multicolumn{6}{|c|}{ Reference category } \\
\hline Asian/Pacific Islander & $1.335 \pm 0.371$ & 3.602 & $<.001$ & $-0.391 \pm 0.310$ & -1.260 & 0.208 \\
\hline Hispanic & $-0.162 \pm 0.529$ & -0.307 & 0.759 & $-0.273 \pm 0.328$ & -0.834 & 0.404 \\
\hline African American & $0.542 \pm 1.132$ & 0.479 & 0.632 & $-0.223 \pm 0.642$ & -0.347 & 0.728 \\
\hline Other & $0.408 \pm 0.517$ & 0.789 & 0.430 & $-0.058 \pm 0.271$ & -0.214 & 0.831 \\
\hline \multicolumn{7}{|l|}{ BMI } \\
\hline Underweight & $0.081 \pm 0.325$ & 0.248 & 0.804 & $-0.278 \pm 0.179$ & -1.551 & 0.121 \\
\hline Normal & \multicolumn{6}{|c|}{ Reference category } \\
\hline Overweight & $0.071 \pm 0.257$ & 0.278 & 0.781 & $-0.384 \pm 0.107$ & -3.571 & $<.001$ \\
\hline Obese & $0.864 \pm 0.317$ & 2.723 & 0.006 & $-0.694 \pm 0.168$ & -4.119 & $<.001$ \\
\hline
\end{tabular}

Freq. of alcohol consumption

Never
Rarely
Occasionally
Regularly
Daily

Last exposure to antibiotics (days)

$$
\begin{aligned}
& -0.802 \pm 0.288 \\
& -0.661 \pm 0.269 \\
& -0.524 \pm 0.287 \\
& -0.608 \pm 0.343
\end{aligned}
$$$$
-2.786
$$$$
-2.456
$$$$
-1.825
$$$$
-1.774
$$

$$
\text { Reference category }
$$

$\begin{array}{llll}0.005 & -0.041 \pm 0.134 & -0.306 & 0.759 \\ 0.014 & -0.104 \pm 0.138 & -0.754 & 0.451 \\ 0.068 & -0.212 \pm 0.144 & -1.470 & 0.142 \\ 0.076 & -0.208 \pm 0.169 & -1.233 & 0.218\end{array}$

$>365$
$<365$
$<180$
$<30$
$<7$

$0.648 \pm 0.246$

\subsection{2}

$1.687 \pm 0.273$

6.188

1.212

$0.568 \pm 0.469$

3.149

Reference category

$\begin{array}{llll}0.008 & -0.136 \pm 0.125 & -1.084 & 0.278 \\ <.001 & 0.115 \pm 0.161 & 0.710 & 0.477 \\ 0.226 & -0.105 \pm 0.263 & -0.401 & 0.689 \\ 0.002 & 0.334 \pm 0.374 & 0.892 & 0.372\end{array}$

Country of residence

USA

UK-Ireland
Europe Continental
Australia and NZL
Canada
Others

$-2.802 \pm 1.037$

$-2.703$

Reference category

Drinking water source

City

Filtered
Bottled
Well

Level of education

$\leq$ High school

$-1.598 \pm 1.380$

$-1.158$

0.007

$0.180 \pm 0.113$

1.590

0.112

$-1.494 \pm 0.982$

$-1.522$

0.247

$0.258 \pm 0.289$

0.892

0.372

0.128

$0.148 \pm 0.227$

0.654

0.513

$-1.470 \pm 0.931$

$-1.578$

0.115

$-0.150 \pm 0.304$

$-0.494$

0.621

$-2.299 \pm 1.083$

$-2.123$

0.034

$0.384 \pm 0.580$

0.661

0.509

Reference category

$$
\begin{aligned}
& 0.094 \pm 0.190 \\
& -0.207 \pm 0.393 \\
& 0.407 \pm 0.295
\end{aligned}
$$

0.493

0.622

$-0.053 \pm 0.094$

$-0.558$

0.577

$-0.527$

0.598

$-0.020 \pm 0.164$

$-0.121$

0.904

1.381

0.167

$0.137 \pm 0.167$

0.822

0.411

$0.957 \pm 0.441$

2.170

0.030

$0.226 \pm 0.239$

0.945

0.344

Reference category

College/bachelor's

$0.176 \pm 0.292$

0.601

0.548

$0.067 \pm 0.111$

0.602

0.547 
Table 4 The outcomes of the logistic and negative binomial components of the fitted ZINB regression model (Continued)

\begin{tabular}{|c|c|c|c|c|c|c|}
\hline \multicolumn{7}{|l|}{ Dog } \\
\hline Absent & \multicolumn{6}{|c|}{ Reference category } \\
\hline True & $0.270 \pm 0.178$ & 1.516 & 0.130 & $-0.044 \pm 0.094$ & -0.469 & 0.639 \\
\hline \multicolumn{7}{|l|}{ C-section } \\
\hline False & \multicolumn{6}{|c|}{ Reference category } \\
\hline True & $0.209 \pm 0.266$ & 0.786 & 0.432 & $0.173 \pm 0.151$ & 1.143 & 0.253 \\
\hline \multicolumn{7}{|l|}{ Vegetable frequency } \\
\hline$<1$ time/week & $0.102 \pm 0.630$ & 0.162 & 0.871 & $-0.191 \pm 0.330$ & -0.580 & 0.562 \\
\hline 1-2 times/week & $0.395 \pm 0.399$ & 0.991 & 0.322 & $-0.099 \pm 0.205$ & -0.485 & 0.628 \\
\hline 3-5 times/week & \multicolumn{6}{|c|}{ Reference category } \\
\hline Daily & $-0.216 \pm 0.288$ & -0.750 & 0.453 & $0.184 \pm 0.114$ & 1.618 & 0.106 \\
\hline \multicolumn{7}{|l|}{ Thyroid condition } \\
\hline No condition & \multicolumn{6}{|c|}{ Reference category } \\
\hline Diagnosed & $-0.093 \pm 0.431$ & -0.216 & 0.829 & $-0.394 \pm 0.166$ & -2.37 & 0.018 \\
\hline
\end{tabular}

We then compared the resilience of the networks to disturbance, using sequential node removal to simulate "attacks" to the networks, an approach previously described [45]. Natural connectivity-the number of closed walks [46]—was used to assess robustness of remaining networks (Fig. 6b). Node removals were performed either by first removing the hub species-nodes with the highest degree (Fig. 6b, left) or betweenness centrality (Fig. 6b, middle)-or at random (Fig. 6b, right). In the degree-based node removal, natural connectivity dropped faster in the $O$. formigenes - network compared to O. formigenes+, indicating that the $O$. formigenes- networks might collapse faster under "ecological attack" when important species were affected.

O. formigenes+ networks also were associated with significantly higher natural connectivity before any nodes were removed (Fig. 6b). To alternatively examine this observation, we performed network inference repeatedly, obtaining a distribution of natural connectivity associated with the O. formigenes+ or $O$. formigenes- networks. The O. formigenes+ networks had significantly higher natural connectivity (Fig. 6c, first panel). Seven other microbial species, whose prevalence and abundance were at the same order of magnitude as $O$. formigenes, were selected as control taxa. Among them, two unclassified species from family Dehalobacterium or rc4-4 showed the contrasting pattern that their presence were associated with networks with lower connectivity; the presence of Rothia mucilaginosa, Rothia dentocariosa, Campylobacter unclassified, cc_115 unclassified, and Neisseria subflava showed the same differential pattern as $O$. formigenes (Fig. 6c).

\section{Discussion}

This study has four major findings:

(i).We confirm extensive variation in O. formigenes relative abundance [35], now with much larger numbers of subjects, indicating a range of approximately $3 \log _{10}$.

(ii). By sequence analysis of the OTUs, we confirmed that humans may be co-colonized by group I and II O. formigenes strains [47], which we mapped to currently described strains [10, 28, 48].

(iii).We confirmed and extended relationships between O. formigenes prevalence and host features, at considerably larger scale, and now with analyses in relation to both $O$. formigenes prevalence and abundance.

(iv).We showed that the host gut microbiota displayed greater community diversity, global network connectivity, and greater resilience to simulated disturbance, in the samples in which $O$. formigenes was detected.

Our data suggest that $O$. formigenes strains resembling strain BLISS, a putative phylogenetic group I strain, might be the most prevalent and abundant strains in the human gastrointestinal tract, while group II $O$. formigenes strains (as exemplified by HOxBLS and OXK) are less common, consistent with a prior study [47]. Knowing which strains are naturally dominant human gut colonizers is important for designing long-term clinical interventions. For example, strain $\mathrm{HC1}$ was administered to primary hyperoxaluria patients, achieving promising shortterm urinary oxalate reduction but failed to maintain 


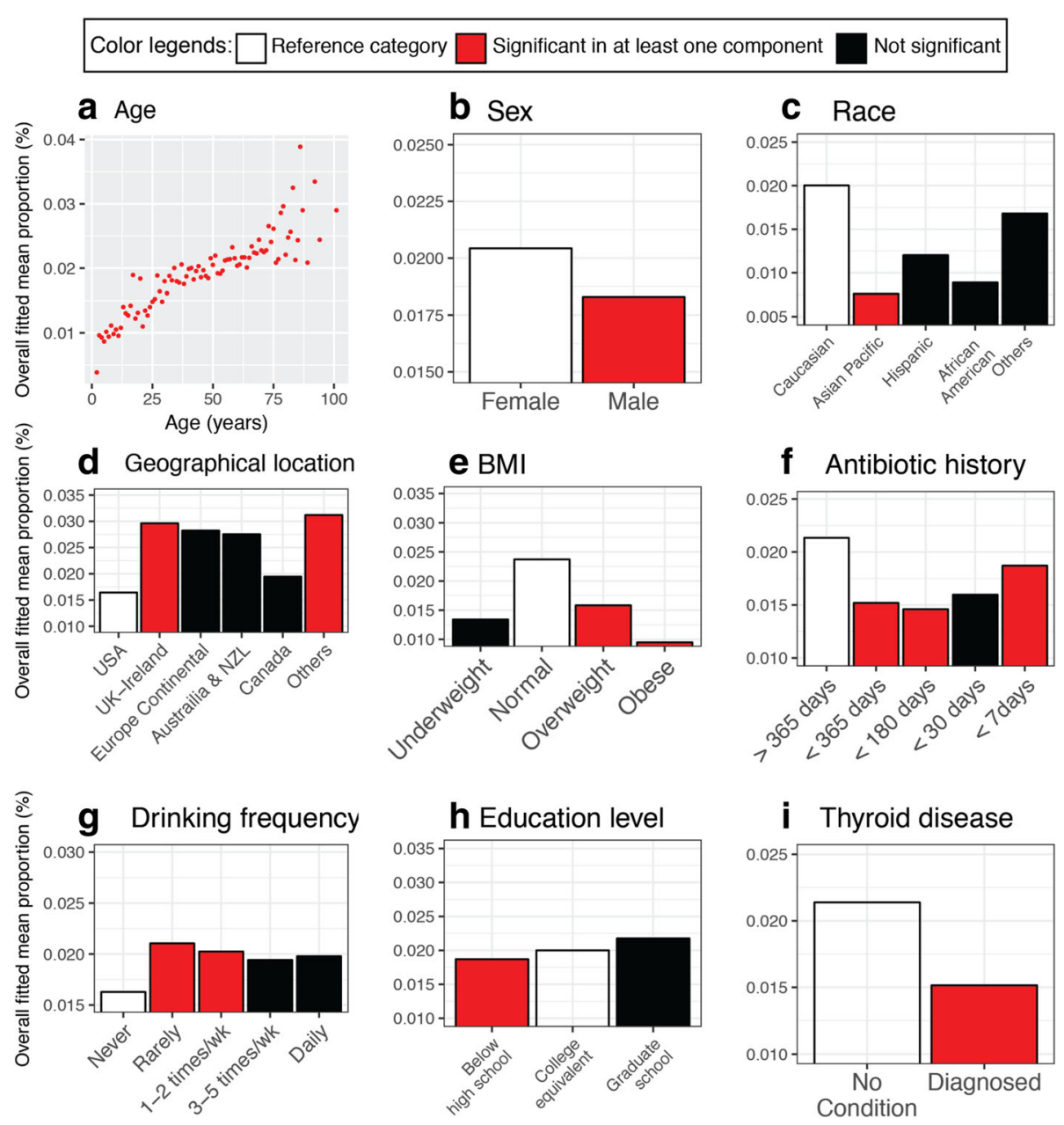

Fig. 3 Predicted relationships between $O$. formigenes abundance and host features by the ZINB model. a-i The overall fitted mean proportions (\%) of $O$. formigenes were plotted as functions of nine significant covariates in the ZINB model fitted with 4945 AGP fecal samples. For each covariate, categories are color-coded by reference (white), not significant (black), or significant in either logistic or negative binomial regression component (red). Panels focus on covariate age (a); sex (b); race (c); geographical location (d); BMI (Underweight, Normal, Overweight and Obese groups are classified based on BMI $\leq 18.5,18.5-25,25.1-30$, and >30, respectively) (e); last exposure to antibiotics (f); alcohol consumption frequency (groups of rarely, occasionally, regularly consuming are defined as a few times/month, 1-2 times/week, and 3-5 times/week) (g); education level (h); and whether has thyroid disease (i)
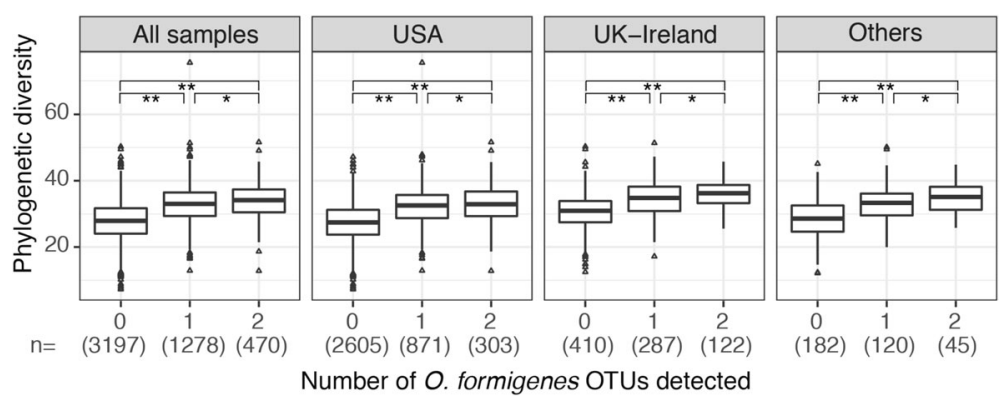

Fig. $4 a$-Diversity measurements in 4945 fecal samples, by number of $O$. formigenes OTUs detected. By Student's $t$ test, ${ }^{*} p$ value $<0.001$ 


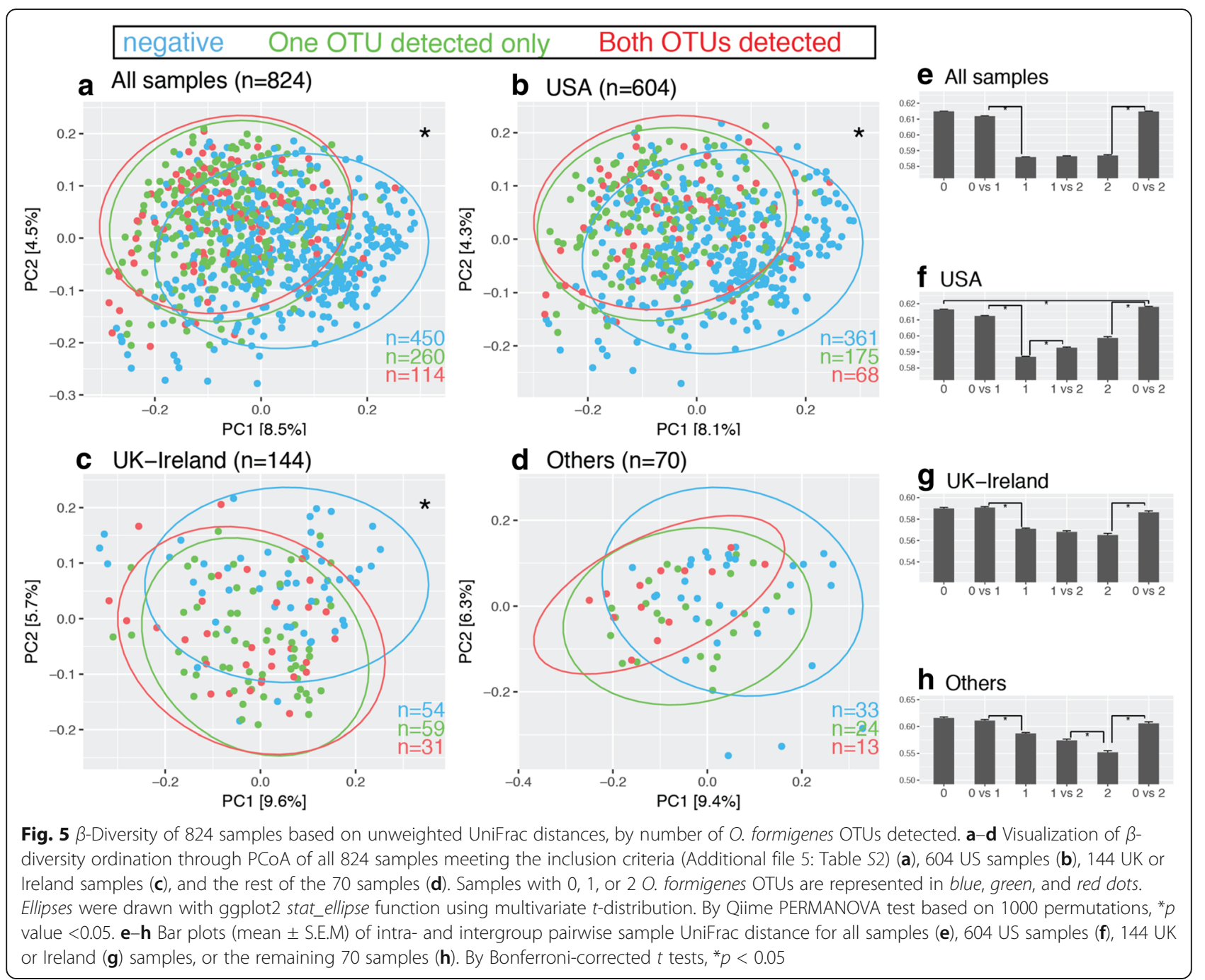

long-term colonization and significant oxalate reduction [17, 20, 21]. All three O. formigenes-associated OTUs we detected showed low $16 \mathrm{~S}$ sequence similarity to $\mathrm{HC1}$ (Additional file 2: Figure S2B), suggesting that it may not be a common human colonizing strain.

Our findings that $O$. formigenes OTUs tend to be co-detected are consistent with our prior work, which used metagenomic data from the Human Microbiome Project [47]. Thus, the shotgun metagenomic and $16 \mathrm{~S}$ analyses provide congruent results, despite the methodological and source population differences. We also now show that those OTUs are detected at elevated abundance when co-present; these observations suggest inter-strain cooperation among $O$. formigenes

Table 5 Microbial species predicted to interact with 0 . formigenes based on three sets of samples

\begin{tabular}{lll}
\hline Taxon & $\begin{array}{l}\text { All } \\
(n=4945)\end{array}$ & $\begin{array}{l}\text { USA } \\
(n=3779)\end{array}$ \\
\hline Bacteria; Firmicutes; Clostridia; Clostridiales; Lachnospiraceae; [Ruminococcus]; gnavus & - & - \\
Bacteria; Firmicutes; Clostridia; Clostridiales; Dehalobacteriaceae; Dehalobacterium; unclassified & - & - \\
$\begin{array}{l}\text { Bacteria; Proteobacteria; Deltaproteobacteria; Desulfovibrionales; Desulfovibrionaceae; Desulfovibrio; } \\
\text { unclassified }\end{array}$ & + & + \\
$\begin{array}{l}\text { Bacteria; Firmicutes; Clostridia; Clostridiales; Christensenellaceae; unclassified; unclassified } \\
\text { Bacteria; Tenericutes; Mollicutes; RF39; unclassified; unclassified; unclassified }\end{array}$ & + \\
$\begin{array}{l}\text { Archaea; Euryarchaeota; Methanobacteria; Methanobacteriales; Methanobacteriaceae; Methanobrevibacter; } \\
\text { unclassified }\end{array}$ & + \\
\hline
\end{tabular}


a

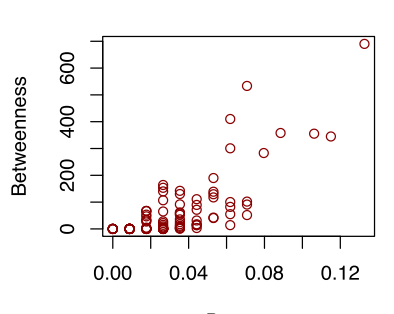

O. formigenes-

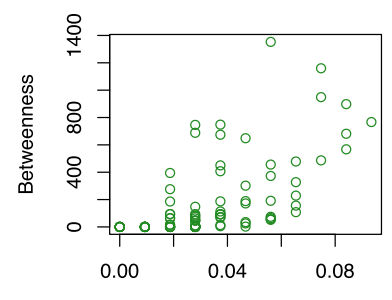

b

Robustness by Degree-based removal

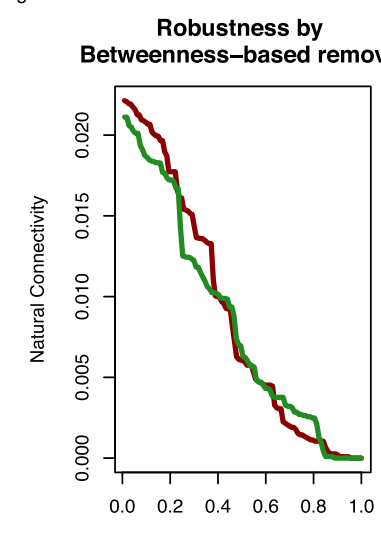

Degree

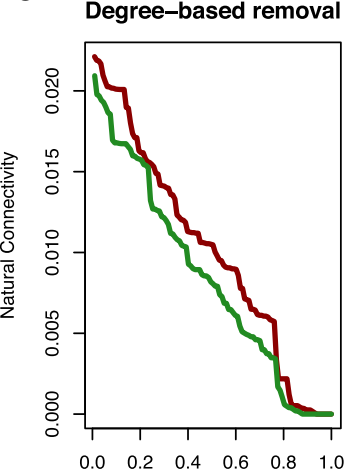

C

Fraction Removed
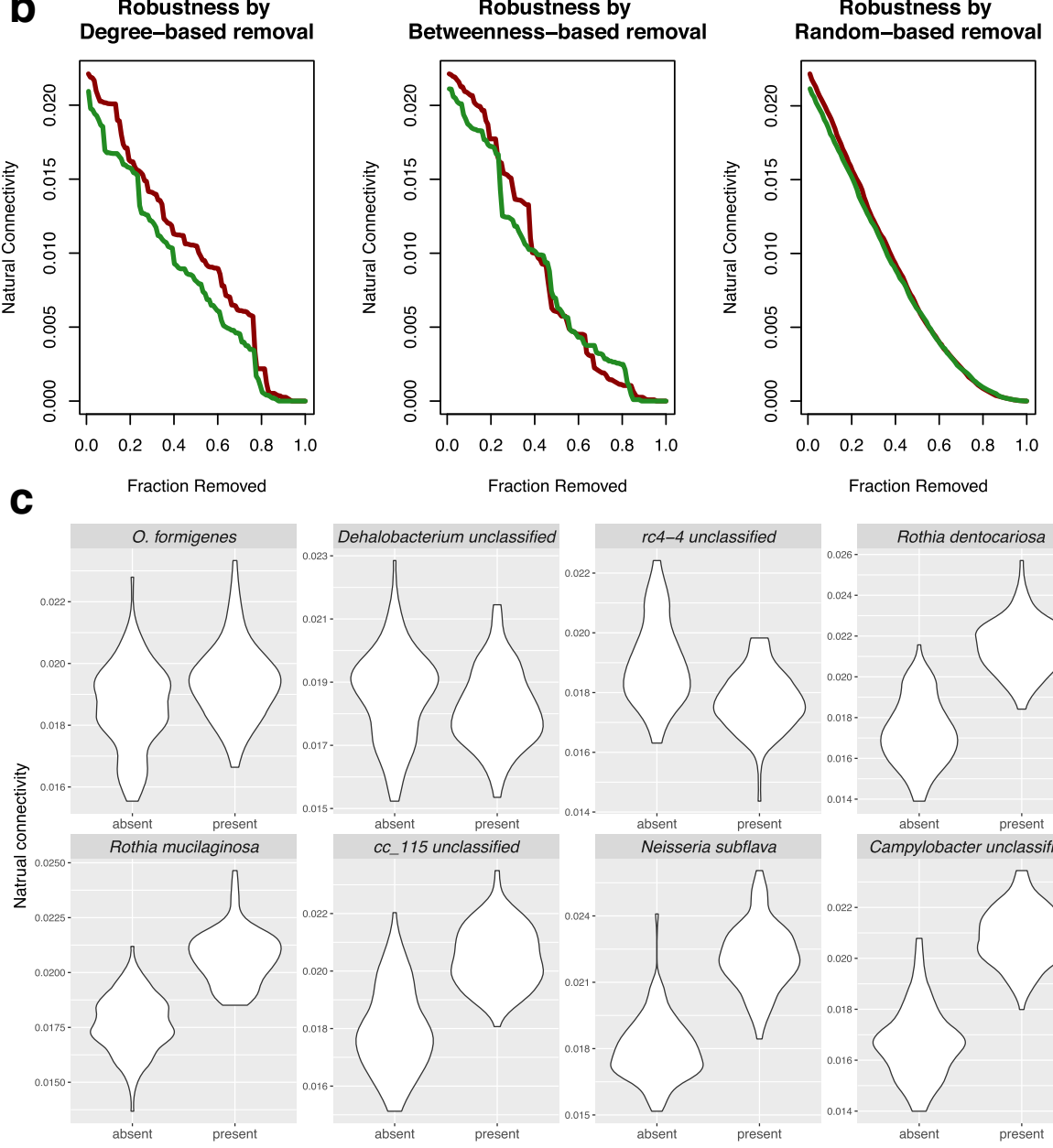

Fraction Removed

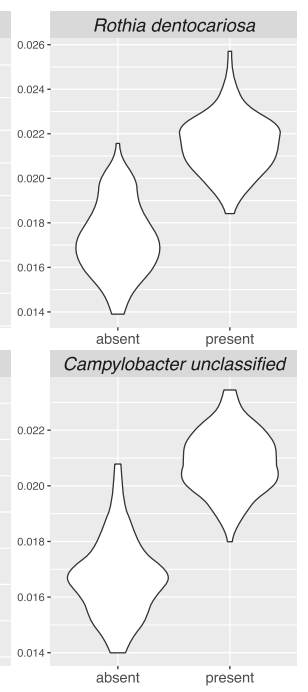

Fig. 6 Microbial structure in relation to O. formigenes colonization. a Centrality of nodes in O. formigenes+ (left) and O. formigenes- (right) networks. The $x$ and $y$ axes represent the normalized node degree and betweenness centrality, respectively. $\mathbf{b}$ Natural connectivity is shown as a function of the remaining size of the network. Node removals were ordered by degree (left), or betweenness centrality (middle), or at random (right). c Natural connectivity associated with presence of $O$. formigenes or with seven other bacterial species as controls. For each category, distribution of natural connectivity was obtained by 100 iterations of network inference with 1000 samples randomly selected per iteration. All comparisons are significant based on Mann-Whitney statistical tests

strains, which needs experimental support. If proven, these findings would suggest that at a clinical level, O. formigenes re-introduction for kidney stone prevention might be facilitated if multiple strains could be administered simultaneously. Alternatively, those observations may reflect cross-feeding, co-aggregation, niche overlap, or use of the same host resource, but without competition. We also cannot rule out technical artifacts, in which $16 \mathrm{~S}$ amplicon sequencing and metagenomic sequencing (with two reference genomes) were both unable to distinguish $O$. formigenes at the strain level and the single taxonomy units that we and others [47] used were mixtures of multiple $O$. formigenes strains. 
The longitudinal analyses showed a considerable proportion of subjects switching $O$. formigenes status. It is unlikely that $O$. formigenes colonization is lost and regained at such high frequency; more likely, limited sequencing depth in the assays underestimates $O$. formigenes presence in samples in which they are present at low abundance. This explanation is supported by our finding that in subjects in whom $O$. formigenes was detected at lower relative levels, detection was more variable (Fig. 2a, middle) than those who are colonized at relatively high levels (Fig. 2a, right). This difference justifies restricting our analysis to samples with $>10,000$ assigned sequences. Better understanding and investigation of $O$. formigenes biology and its therapeutic applications may require deeper sequencing and more curated reference genomes. These limitations will be relevant to approaches for other low abundant taxa of potential medical interest.

Prior smaller studies have linked O. formigenes prevalence with host age, sex, location, education, race/ethnicity, and oxalate consumption [31, 32, 48]. Our multivariate analysis further interrogated the underlying relationships, dealing with the technical issue of microbial data sparsity using a robust statistical model $[49,50]$. Our observations that males carry $O$. formigenes at significantly lower abundance than females (Fig. 3b) correlate with the unexplained twofold higher kidney stone incidence in males compared to females $[4,51]$.

Prior studies have identified oxalate deposition within the thyroid gland in humans and other mammals [52-62]. A study of healthy thyroid tissues obtained at autopsy from 182 individuals found a negative correlation between spatial distributions of calcium oxalate crystals and triiodothyronine (T3)-producing colloids [59], suggesting that oxalate precipitation may be inversely related to normal thyroid function. Our observations that subjects diagnosed with thyroid diseases were colonized by $O$. formigenes at lower prevalence (Table 3) and abundance (Fig. 3i) are consistent with the hypothesis that $O$. formigenes may be beneficial to host thyroid function by lowering circulating oxalate. However, the sample size of AGP subjects with $(n=363)$ or without $(n=2490)$ thyroid disease is unbalanced, and the AGP includes no information of specific type of thyroid abnormalities. Thus, future targeted studies are needed to further examine this hypothesis.

That $O$. formigenes detection is correlated with higher phylogenetic diversity in the host microbiota (Fig. 4) is consistent with comparisons of US subjects and Amerindian hunter gatherers [63]. The Amerindians had significantly higher diversity and nearly universal $O$. formigenes colonization at high abundance; $O$. formigenes was one of the most differentiating taxa between those two populations [63]. These results may indicate that a more diverse gut microbial community has greater likelihood of harboring $O$. formigenes or alternatively could reflect technical issues such as differences in sequencing depth.

If the prevalence and abundance of $O$. formigenes is indeed an indicator of the ecological state of the microbiota, then the disappearing microbiota theory [64] might predict association with particular disease states that have risen while we have been losing diversity. Of interest to us are the recent studies describing rising prevalence of kidney stones in the USA [51] and other countries [65-67]. Furthermore, the linkage observed of O. formigenes absence to both obesity (Fig. 3e) and thyroid disease (Fig. 3i) is consistent with that hypothesis. Our analysis of inferred ecological networks also suggests that $O$. formigenes presence may be one indicator of host microbiota integrity and ultimately may be a marker for host physiology.

\section{Conclusions}

In conclusion, evidence is growing that $O$. formigenes has medical significance in humans $[4,5,13,15,30,32]$. We show that the AGP is a valuable resource and present a systematic framework to explore the biology of O. formigenes in humans. The relationships that we observed illustrate the power of a crowd-sourced enterprise, if done on sufficient scale, to answer biological and medical questions. Nevertheless, the observations we report should be considered as hypothesis-generating; carefully designed experiments are needed to establish the underlying causal relationships and to help design targeted clinical interventions. Nevertheless, the systematic framework we built can be extended to study other bacterial taxa of interest.

\section{Methods \\ Data acquisition and processing}

In the AGP, samples were self-obtained by study participants using sample kits containing detailed instructions [68] and then shipped by mail to the AGP home lab, accompanied by completion of metadata questionnaires. Sample DNA extraction, library preparation, and sequencing were performed as described [69]. The AGP consortium processed data with closed-reference OTU-picking pipeline [70]. We also performed open-reference OTU picking using Uclust [22] to search for novel O. formigenes-associated OTUs. In our study, the raw sequences [71] and the closed-reference OTU-picking results [72] were directly downloaded from the ftp website and the metadata were shared by the AGP. All those data could also be alternatively acquired via the Qiita [73] website under study number 10317. 
Phylogenetic analysis of $O$. formigenes strains and detected OTUs

Complete 16S rRNA gene sequences of 13 O. formigenes strains, three O. formigenes-associated OTUs, and Oxalobacter vibrioformis were downloaded from the Greengenes website [24]. The V4 region was a subset based on the primers used for the AGP (FWD:GTGY CAGCMGCCGCGGTAA, REV:GGACTACNVGGGTW TCTAAT; 515FB-806RB) [69]. Phylogenetic trees were constructed with the sequences of the complete $16 \mathrm{~S}$ (Additional file 2: Figure S2A) or V4 region only (Fig. 1b) via phylogeny.fr [74]. Four steps were performed: (i) initial alignment by MUSCLE 3.8.1, (ii) alignment refinement by Gblock, (iii) maximum likelihood phylogeny analysis by PhyML, and (iv) tree rendering by TreeDyn [75-80]. For detailed methods and parameters, refer to phylogene.fr website documentation section 2.1 [81]. The sequence identity matrix (Additional file 2: Figure S2B) was calculated with $(1-$ dist)\% by the dist.alignment function [82] from the seqinr package in R, based on the alignment results that were obtained during the $16 \mathrm{~S} \mathrm{~V} 4$ region sequence alignment for Fig. 1b.

\section{Sample diversity analyses}

Intra-sample $\alpha$-diversity was calculated using QIIME, using four metrics, phylogenetic diversity [37], Shannon index [39], Chao1 index [38], and observed number of OTUs, at rarefaction depths from 1000 to 10,000 sequences/sample. Pairwise inter-sample $\beta$-diversity was calculated using the unweighted UniFrac distance metric [23] and Bray-Curtis dissimilarities [41].

\section{Multivariate analysis}

(i) Description of the multivariate model: A multiple zero-inflated negative binomial regression (ZINB) [33] model was used for the differential abundance analysis on $O$. formigenes, to handle its excessive zero abundances in its read count (64\%) and the overdispersion (the mean 5.7 is much smaller than the variance 284.4). The ZINB model consists of two different components, a logistic regression for modeling the excessive zeros and a negative binomial regression for modeling the remaining count values. To adjust for the varying number of total read counts, both components of the ZINB model included the $\log$ (total read counts) as the offset variable such that the ZINB model assesses the proportions of $O$. formigenes rather than the count [35]. The canonical link functions were used with logit for the logistic regression and $\log$ for the negative binomial regression. Missing data in each categorical variable was included into a separated hidden category [83]. (ii) Variable selection: The variables were selected based on prior literature, data availability and quality, biological relevance, and investigators' interest yielding 14 variables to be included in the analysis (Table 1). Among those, age, sex, and race were included as the baseline covariates to both the logistic and the negative binomial regression components. The other covariates were subsequently added to the regressions if they had univariate $p$ value $<0.1$ either in logistic or negative binomial regression model (Table 1 ). The criterion $p$ value $<0.1$ was used, which is less stringent than the canonical criterion $p<0.05$, in considering that some covariates that are weak predictors in univariate analyses might be influential when in combination with other covariates. (iii) Model selection: We compared the performance of the ZINB model with zero-inflated Poisson (ZIP) [49], in terms of fitness to the data based on the same link functions, offset variable, and covariates. The ZINB model outperformed the ZIP model with smaller (a) Akaike information criterion (AIC) [84] [ZINB 18,177 vs ZIP 41,456], (b) Bayesian information criterion (BIC) [85] [ZINB 18,765 vs ZIP 42,035], and (c) log likelihood [ZINB -8997.6 (DF 91) vs ZIP -20,637.4 (DF 90); $p<0.001$ ] by the likelihood ratio test, suggesting that the goodness-of-fit was significantly improved in ZINB over ZIP. (iv) Overall fitted mean proportions (\%): Overall fitted mean proportions were calculated by the average predicted value (APV) method [35], which is predicted $O$. formigenes count values divided by the mean total read counts under each exposure status, as measurements to estimate the effect direction and magnitude on $O$. formigenes relative abundance.

\section{Construction of microbial association networks}

Network was constructed at species level by summing up all associated OTUs for each species. Species that were present in at least $20 \%$ of the samples were selected for network inference by SPIEC-EASI [42]. The default setting of SPIEC-EASI accepts absolute abundance of taxa as input and applies centered log-ration transformation to eliminate the unit-sum constraint of data [86]. Networks were constructed with the SPIEC-EASI [42] package in $\mathrm{R}$ in neighborhood selection mode with parameters set as method $=$ "mb," sel.criterion = "bstars," lambda.min.ratio $=2 \mathrm{e}^{-1}$, nlambda $=100$, pulsar.params $=$ list(rep.num $=20$, ncores $=2$ ). The species-species interacting directions were predicted based on the average coefficients calculated via the beta matrix. Edge centrality and betweenness centrality were calculated using degree and centralization.betweenness functions with the igraph in $\mathrm{R}$, normalized against the theoretical maximum. Natural connectivity, a variant of the Estrada index [46], was calculated as described [46], through the following equation:

$$
\left.\log \left(\sum e^{x}\right)\right) /(N-\log (N))
$$

where $N$ is the number of nodes in the network and $x$ is 
the average of eigenvalues of the graph adjacency matrix, which was calculated using get.adjacency and eigen $\mathrm{R}$ functions.

The distributions of natural connectivity [46] associated with $O$. formigenes+ and O. formigenes- networks were acquired from 100 iterations of network inference, randomly selecting $1000 \mathrm{O}$. formigenes+ and O. formigenes- samples in each iteration. Seven control species were selected based on their similarity to O. formigenes population parameters: detection rates of $30-40 \%$ and relative abundances of $10^{-2}-10^{-3}$. The distributions of natural connectivity associated with presence or absence of the control species were calculated the same way as with $O$. formigenes.

\section{Additional files}

Additional file 1: Figure S1. Workflow of American Gut Project data processing. The approaches of open- and closed-reference picking are shown, with the accompanying statistics on sequence numbers. (PDF $34 \mathrm{~kb}$ )

Additional file 2: Figure S2. Phylogenetic analyses of $O$. formigenes strains and OTUs detected. Panel A. Maximum likelihood O. formigenes phylogenetic tree. The tree was built from full-length 16S rRNA gene sequences of $O$. formigenes strains [group I, purple; group II, green and strain BLISS (group unknown), in black; and O. vibrioformis selected from the Oxalobacter family], downloaded from Greengenes. The log-likelihood of tree is -3191 .23. Branch support values are designated in red. Statistical details of the tree are included in the Additional files 11 and 12. Panel B. Sequence similarity matrix using $16 \mathrm{~S} \mathrm{~V} 4$ region of $13 \mathrm{O}$. formigenes strains, O. vicrioformis, and 3 OTUs. Refer to the "Methods" section for the calculation of similarity. (PDF $600 \mathrm{~kb}$ )

Additional file 3: Figure S3. Sequencing depth in relation to the number of $O$. formigenes OTUs present in a sample. Panels $A$, B. Panels focus on fecal samples with over 1000 (panel A) or 10,000 (panel B) sequences per sample, considering OTUs 7366 and 360508 . Fold changes were calculated using the median sequencing depths of each group. (PDF $250 \mathrm{~kb}$ )

Additional file 4: Figure S4. Number of study subjects, by the number of samples provided. (PDF $25 \mathrm{~kb}$ )

Additional file 5: Table S1. Prevalence of O. formigenes in 4945 AGP fecal samples, by locality of birth and current residence. Table $\mathbf{S 2}$. Inclusion criteria for individuals whose samples were used in the $\beta$ diversity (Fig. 5) analyses. (DOCX 19 kb)

Additional file 6: Figure S5. Correlation between diet and $O$. formigenes relative abundance in 197 subjects. Panels focus on oxalate (left), calcium (middle), or oxalate/calcium (right). Dietary intake over the 90 days preceding sample collection was estimated through the Vioscreen questionnaire. ${ }^{*} p$ value $<0.05$, by Spearman rank correlation. (PDF $96 \mathrm{~kb}$ )

Additional file 7: Figure S6. $a$-Diversity measurements in 4945 fecal samples, by number of $O$. formigenes OTUs detected. The number of samples is indicated in parentheses. Rarefaction depths from 1000 to 10,000 segs/sample are shown. All comparisons are significant at 10,000 seqs/sample, Bonferroni-corrected nonparametric two-sample $t$ tests with 999 Monte Carlo permutations. (PDF $285 \mathrm{~kb}$ )

Additional file 8: Figure S7. $\beta$-Diversity of 824 samples based on BrayCurtis dissimilarities, by number of $O$. formigenes OTUs detected. Panels A-D. Visualization of $\beta$-diversity ordination through PCoA of all 824 samples meeting the inclusion criteria (Additional file 5: Table S2) (A), 604 US samples (B), 144 UK or Ireland samples (C), the rest of the 70 samples (D). Samples with 0, 1, or 2 O. formigenes OTUs are represented in blue, green, and red dots. Ellipses were drawn with ggplot2 stat_ellipse function using multivariate $t$-distribution. By Adonis test, ${ }^{*} p$ value $<0.05$.
Panels E-H. Bar plots (mean \pm S.E.M) of intra- and intergroup pairwise sample distance by Bray-Curtis dissimilarities for all (E), 604 US (F), and 144 UK or Ireland (G) samples or for the remaining 70 samples (H). By Bonferroni-corrected $t$ tests, ${ }^{*} p<0.05$. (PDF $158 \mathrm{~kb}$ )

Additional file 9: Alignment results of $16 \mathrm{~S}$ rRNA gene $\mathrm{V} 4$ region sequence of $13 \mathrm{O}$. formigenes strains, $3 \mathrm{O}$. formigenes-associated OTUs, and O. vicrioformis. (TXT $14 \mathrm{~kb}$ )

Additional file 10: Statistical details of the phylogenetic tree in Fig. 1b. (TXT 424 bytes)

Additional file 11: Alignment results of full 16S rRNA gene sequences of $13 \mathrm{O}$. formigenes strains and O. vicrioformis. (FASTA $22 \mathrm{~kb}$ )

Additional file 12: Statistical details of the phylogenetic tree in Additional file 2: Figure S2A. (FASTA 424 bytes)

Additional file 13: $R$ script for multivariate analysis. ( $R 33 \mathrm{~kb}$ )

Additional file 14: $R$ script for network analysis. ( $\mathrm{R} 9 \mathrm{~kb}$ )

Additional file 15: $R$ functions that were used in Additional files 13 and 14 . (R $2 \mathrm{~kb})$

\section{Abbreviations}

AGP: American Gut Project; Gl tract: Gastrointestinal tract; OTUs: Operational taxonomic units; ZINB: Zero-inflated negative binomial model; ZIP: Zeroinflated Poisson model; SPIEC-EASI: Sparse Inverse Covariance Estimation for Ecological Association and Statistical Inference

\section{Acknowledgements}

This work has utilized computing resources at the High-Performance Computing Facility at NYU Langone Medical Center. We thank Rob Knight and Embriete Hyde from AGP for the useful advice and thank Christian Mueller, Steven Daniels, Kelly Ruggles, Stuart Brown, and Aristotellis Tsirigos for their insights.

\section{Funding}

This study was supported in part by U01A1122285 and R01DK090989 and R01DK110014 from the National Institutes of Health and by the C \& D, Belfer, and Knapp Family Funds and the Saul Farber scholarship. The authors also gratefully acknowledge support of the Rare Kidney Stone Consortium (U54KD083908), a part of NIH Rare Diseases Clinical Research Network (RDCRN), funded by the NIDDK and the National Center for Advancing Translational Sciences.

\section{Availability of data and materials}

The data used in the present study can be accessed through the Qiita website under study number 10317 [https://qiita.ucsd.edu/study/ description/10317] and downloaded via the following link [https:// qiita.ucsd.edu/download_study_bioms/10317]. The R scripts used for the multivariate analysis and the network analysis are included in Additional files 13,14 and 15

\section{Authors' contributions}

$M J B, M L$, and $L N$ designed the study. ML, HK, ZDK, HL, and TB performed all the data analysis with $\mathrm{HK}$ and $\mathrm{HL}$ focused on the multivariate analysis, ZDK focused to the network analysis, and TB helped process the raw data. MJB, $M L, L N, H K$, and $A P$ interpreted the analysis results. $M L$ and $M J B$ wrote the manuscript. All authors contributed to and reviewed the final manuscript. All authors read and approved the final manuscript.

Ethics approval and consent to participate

Not applicable.

\section{Consent for publication}

Not applicable.

\section{Competing interests}

The authors declare that they have no competing interests. 


\section{Publisher's Note}

Springer Nature remains neutral with regard to jurisdictional claims in published maps and institutional affiliations.

\begin{abstract}
Author details
'Sackler Institute of Graduate Biomedical Sciences, New York University School of Medicine, New York, NY 10016, USA. ${ }^{2}$ Department of Population Health, New York University School of Medicine, New York, NY 10016, USA. ${ }^{3}$ Department of Microbiology, New York University School of Medicine, New York, NY 10016, USA. ${ }^{4}$ Department of Medicine, New York University School of Medicine, New York, NY 10016, USA. ${ }^{5}$ Medical Service, New York Harbor Department of Veterans Affairs Medical Center, New York, NY 10010, USA
\end{abstract}

Received: 30 May 2017 Accepted: 25 July 2017

Published online: 25 August 2017

\section{References}

1. Holmes RP, Ambrosius WT, Assimos DG. Dietary oxalate loads and renal oxalate handling. J Urol. 2005;174:943-7. discussion 947.

2. Holmes RP, Assimos DG. Glyoxylate synthesis, and its modulation and influence on oxalate synthesis. J Urol. 1998;160:1617-24.

3. Simpson $\mathrm{GL}$, Ortwerth BJ. The non-oxidative degradation of ascorbic acid at physiological conditions. Biochim Biophys Acta. 2000;1501:12-24.

4. Brzica H, Breljak D, Burckhardt BC, Burckhardt G, Sabolic I. Oxalate: from the environment to kidney stones. Arh Hig Rada Toksikol. 2013;64:609-30.

5. Chai W, Liebman M, Kynast-Gales S, Massey L. Oxalate absorption and endogenous oxalate synthesis from ascorbate in calcium oxalate stone formers and non-stone formers. Am J Kidney Dis. 2004:44:1060-9.

6. Siener R, Schade N, Nicolay C, von Unruh GE, Hesse A. The efficacy of dietary intervention on urinary risk factors for stone formation in recurrent calcium oxalate stone patients. J Urol. 2005;173:1601-5.

7. Verbruggen LA, Bourgain C, Verbeelen D. Late presentation and microcrystalline arthropathy in primary hyperoxaluria. Clin Exp Rheumatol. 1989;7:631-3.

8. Sarraf P, Kay J, Reginato AM. Non-crystalline and crystalline rheumatic disorders in chronic kidney disease. Curr Rheumatol Rep. 2008;10:235-48.

9. Castellaro AM, Tonda A, Cejas HH, Ferreyra H, Caputto BL, Pucci OA, Gil GA. Oxalate induces breast cancer. BMC Cancer. 2015;15:761.

10. Allison MJ, Dawson KA, Mayberry WR, Foss JG. Oxalobacter formigenes gen. nov., sp. nov.: oxalate-degrading anaerobes that inhabit the gastrointestinal tract. Arch Microbiol. 1985;141:1-7.

11. Miller AW, Dearing D. The metabolic and ecological interactions of oxalatedegrading bacteria in the mammalian gut. Pathogens. 2013;2:636-52.

12. Azcarate-Peril MA, Bruno-Barcena JM, Hassan HM, Klaenhammer TR. Transcriptional and functional analysis of oxalyl-coenzyme A (CoA) decarboxylase and formyl-CoA transferase genes from lactobacillus acidophilus. Appl Environ Microbiol. 2006;72:1891-9.

13. Arvans D, Jung YC, Antonopoulos D, Koval J, Granja I, Bashir M, Karrar E, Roy-Chowdhury J, Musch M, Asplin J, et al. Oxalobacter formigenes-derived bioactive factors stimulate oxalate transport by intestinal epithelial cells. J Am Soc Nephrol. 2017;28:876-87.

14. Sidhu H, Schmidt M, Cornelius J, Thamilselvan S, Khan S, Hesse A, Peck A. Direct correlation between hyperoxaluria/oxalate stone disease and the absence of the gastrointestinal tract-dwelling bacterium Oxalobacter formigenes: possible prevention by gut recolonization or enzyme replacement therapy. J Am Soc Nephrol. 1999;10:S334-40.

15. Duncan SH, Richardson AJ, Kaul P, Holmes RP, Allison MJ, Stewart CS. Oxalobacter formigenes and its potential role in human health. Appl Environ Microbiol. 2002;68:3841-7.

16. Stewart CS, Duncan SH, Cave DR. Oxalobacter formigenes and its role in oxalate metabolism in the human gut. FEMS Microbiol Lett. 2004;230:1-7.

17. Hoppe B, von Unruh G, Laube N, Hesse A, Sidhu H. Oxalate degrading bacteria: new treatment option for patients with primary and secondary hyperoxaluria? Urol Res. 2005;33:372-5.

18. Hatch M, Cornelius J, Allison M, Sidhu H, Peck A, Freel RW. Oxalobacter sp. reduces urinary oxalate excretion by promoting enteric oxalate secretion. Kidney Int. 2006;69:691-8.

19. Hatch M, Freel RW. A human strain of Oxalobacter (HC-1) promotes enteric oxalate secretion in the small intestine of mice and reduces urinary oxalate excretion. Urolithiasis. 2013;41:379-84.
20. Hoppe B, Groothoff JW, Hulton SA, Cochat P, Niaudet P, Kemper MJ Deschenes G, Unwin R, Milliner D. Efficacy and safety of Oxalobacter formigenes to reduce urinary oxalate in primary hyperoxaluria. Nephrol Dial Transplant. 2011:26:3609-15.

21. Hoppe B, Niaudet P, Salomon R, Harambat J, Hulton SA, Van't Hoff W, Moochhala SH, Deschenes G, Lindner E, Sjogren A, Cochat P. A randomised phase $1 / I I$ trial to evaluate the efficacy and safety of orally administered Oxalobacter formigenes to treat primary hyperoxaluria. Pediatr Nephrol. 2017:32:781-90

22. Caporaso JG, Kuczynski J, Stombaugh J, Bittinger K, Bushman FD, Costello EK, Fierer N, Pena AG, Goodrich JK, Gordon JI. QIIME allows analysis of highthroughput community sequencing data. Nat Methods. 2010;7:335-6.

23. Hamady M, Lozupone C, Knight R. Fast UniFrac: facilitating high-throughput phylogenetic analyses of microbial communities including analysis of pyrosequencing and PhyloChip data. ISME J. 2010;4:17-27.

24. DeSantis TZ, Hugenholtz P, Larsen N, Rojas M, Brodie EL, Keller K, Huber T, Dalevi D, Hu P, Andersen GL. Greengenes, a chimera-checked 165 rRNA gene database and workbench compatible with ARB. Appl Environ Microbiol. 2006:72:5069-72

25. Jensen NS, Allison MJ. Studies on the diversity among anaerobic oxalatedegrading bacteria now in the species Oxalobacter formi- genes. In Abstracts of the 94th General Meeting of the American Society for Microbiology 1994. Abstr. I-12. Washington, D.C: American Society for Microbiology; 1994. p. 255

26. Sidhu $\mathrm{H}$, Allison M, Peck A. Identification and classification of Oxalobacter formigenes strains by using oligonucleotide probes and primers. J Clin Microbiol. 1997;35:350-3.

27. The Greengenes database: Oxalobacter formigenes str. BLISS. http:// greengenes.lbl.gov/cgi-bin/show_one_record_v2.pl?prokMSA_id=7366. Accessed 15 Apr 2017.

28. Oxalobacter formigenes str. BLISS 165 ribosomal RNA gene sequence at National Center for Biotechnology Information. 1998. https://www.ncbi.n/m. nih.gov/nuccore/U49750.1. Accessed 15 Apr 2017.

29. Calculate statistical significance of the overlap between two groups. http:// nemates.org/MA/progs/overlap_stats.html. Accessed 15 Arp 2017.

30. Kaufman DW, Kelly JP, Curhan GC, Anderson TE, Dretler SP, Preminger GM Cave DR. Oxalobacter formigenes may reduce the risk of calcium oxalate kidney stones. J Am Soc Nephrol. 2008;19:1197-203.

31. Kelly JP, Curhan GC, Cave DR, Anderson TE, Kaufman DW. Factors related to colonization with Oxalobacter formigenes in U.S. adults. J Endourol. 2011;25: $673-9$.

32. Mittal RD, Kumar R, Bid HK, Mittal B. Effect of antibiotics on Oxalobacter formigenes colonization of human gastrointestinal tract. J Endourol. 2005;19:102-6.

33. Agresti A. Foundations of linear and generalized linear models. Hoboken: John Wiley \& Sons; 2015.

34. Preisser JS, Stamm JW, Long DL, Kincade ME. Review and recommendations for zero-inflated count regression modeling of dental caries indices in epidemiological studies. Caries Res. 2012;46:413-23.

35. Albert JM, Wang W, Nelson S. Estimating overall exposure effects for zeroinflated regression models with application to dental caries. Stat Methods Med Res. 2014;23:257-78.

36. The VioScreen questionnaire website. http://www.viocare.com/vioscreen. html. Accessed 27 Apr 2017

37. Faith DP. Conservation evaluation and phylogenetic diversity. Biol Conserv. 1992;61:1-10.

38. Chao A. Nonparametric estimation of the number of classes in a population Scand J Stat. 1984:11:265-70

39. Lin J. Divergence measures based on the Shannon entropy. IEEE Trans Inf Theory. 1991;37:145-51.

40. Lozupone C, Knight R. UniFrac: a new phylogenetic method for comparing microbial communities. Appl Environ Microbiol. 2005;71:8228-35.

41. Bray JR, Curtis JT. An ordination of the upland forest communities of southern Wisconsin. Ecol Monogr. 1957;27:325-49.

42. Kurtz ZD, Müller CL, Miraldi ER, Littman DR, Blaser MJ, Bonneau RA. Sparse and compositionally robust inference of microbial ecological networks. PLoS Comput Biol. 2015;11:e1004226.

43. Hakimi SL. On realizability of a set of integers as degrees of the vertices of a linear graph. I. J Soc Ind Appl Math. 1962;10:496-506.

44. Freeman LC. A set of measures of centrality based on betweenness. Sociometry. 1977;40:35-41. 
45. Mahana D, Trent CM, Kurtz ZD, Bokulich NA, Battaglia T, Chung J, Muller CL, $\mathrm{Li} \mathrm{H}$, Bonneau RA, Blaser MJ. Antibiotic perturbation of the murine gut microbiome enhances the adiposity, insulin resistance, and liver disease associated with high-fat diet. Genome Med. 2016;8:48.

46. Wu J, Mauricio B, Tan Y-J, Deng H-Z. Natural connectivity of complex networks. Chin Phys Lett. 2010;27:078902.

47. Barnett C, Nazzal L, Goldfarb DS, Blaser MJ. The presence of Oxalobacter formigenes in the microbiome of healthy young adults. J Urol. 2016;195: 499-506.

48. Knight J, Deora R, Assimos DG, Holmes RP. The genetic composition of Oxalobacter formigenes and its relationship to colonization and calcium oxalate stone disease. Urolithiasis. 2013;41:187-96.

49. Lambert D. Zero-inflated Poisson regression, with an application to defects in manufacturing. Technometrics. 1992;34:1-14.

50. Meinshausen N, Bühlmann P. High-dimensional graphs and variable selection with the lasso. Ann Stat. 2006;34:1436-62.

51. Soucie JM, Thun MJ, Coates RJ, McClellan W, Austin H. Demographic and geographic variability of kidney stones in the United States. Kidney Int. 1994;46:893-9.

52. Furuta M, Ozaki M. Calcium oxalate crystals in the kidney and thyroid of leprosy patients. Int J Lepr Other Mycobact Dis. 1970;38:286-93.

53. Chaplin AJ. Some observations on the demonstration of calcium oxalate in tissue sections. Stain Technol. 1974:49:165-73.

54. Goldman M, Doering GJ. The effect of dietary ingestion of oxalic acid on thyroid function in male and female Long-Evans rats. Toxicol Appl Pharmacol. 1979;48:409-14.

55. Reid JD, Choi CH, Oldroyd NO. Calcium oxalate crystals in the thyroid. Their identification, prevalence, origin, and possible significance. Am J Clin Pathol. 1987;87:443-54

56. Hackett RL, Khan SR. Presence of calcium oxalate crystals in the mammalian thyroid gland. Scanning Microsc. 1988;2:241-6.

57. Reid JD. Calcium oxalate in mammalian thyroids: a re-evaluation. J Comp Pathol. 1991;105:109-15.

58. Wahl R, Fuchs R, Kallee E. Oxalate in the human thyroid gland. Eur J Clin Chem Clin Biochem. 1993;31:559-65.

59. Katoh R, Kawaoi A, Muramatsu A, Hemmi A, Suzuki K. Birefringent (calcium oxalate) crystals in thyroid diseases. A clinicopathological study with possible implications for differential diagnosis. Am J Surg Pathol. 1993;17: 698-705.

60. Katoh R, Suzuki K, Hemmi A, Kawaoi A. Nature and significance of calcium oxalate crystals in normal human thyroid gland. A clinicopathological and immunohistochemical study. Virchows Arch A Pathol Anat Histopathol. 1993;422:301-6.

61. Shimizu M, Hirokawa M, Kanahara T, Manabe T. Calcium oxalate crystals in thyroid fine needle aspiration cytology. Acta Cytol. 1999;43:575-8.

62. Pusztaszeri M. Birefringent crystals in thyroid fine-needle aspiration cytology. Diagn Cytopathol. 2016;44:814-5.

63. Clemente JC, Pehrsson EC, Blaser MJ, Sandhu K, Gao Z, Wang B, Magris M, Hidalgo G, Contreras M, Noya-Alarcón Ó, Lander O. The microbiome of uncontacted Amerindians. Science advances. 2015;1(3):e1500183.

64. Blaser MJ. Who are we? Indigenous microbes and the ecology of human diseases. EMBO Reports. 2006;7:956.

65. Yasui T, Iguchi M, Suzuki S, Kohri K. Prevalence and epidemiological characteristics of urolithiasis in Japan: national trends between 1965 and 2005. Urology. 2008;71:209-13.

66. Trinchieri A, Coppi F, Montanari E, Del Nero A, Zanetti G, Pisani E. Increase in the prevalence of symptomatic upper urinary tract stones during the last ten years. Eur Urol. 2000;37:23-5.

67. Hesse A, Brandle E, Wilbert D, Kohrmann KU, Alken P. Study on the prevalence and incidence of urolithiasis in Germany comparing the years 1979 vs. 2000. Eur Urol. 2003:44:709-13.

68. The American Gut Project sample collection instructions. https://www. microbio.me/AmericanGut/static/img/full_instructions.pdf. Accessed 12 Dec 2016.

69. Earth microbiome project $16 \mathrm{~S}$ Illumina amplicon protocal. http://press.igsb. anl.gov/earthmicrobiome/protocols-and-standards/16s/. Accessed $12 \mathrm{Apr}$ 2017.

70. The American Gut Project data analysis pipeline. http://nbviewer.jupyter.org/ github/biocore/American-Gut/blob/master/ipynb/module2_v1.0.ipynb. Accessed 12 Dec 2016.
71. The American Gut Project sequencing reads. ftp://ftp.microbio.me/ AmericanGut/ag-May-31-2016/01_raw/sequences.fna. Accessed 12 Dec 2016.

72. The American Gut Project closed-reference OTU-picking biom table. ftp:// ftp.microbio.me/AmericanGut/ag-May-31-2016/03-otus/notrim/gg-13_8-97percent/otu_table.biom. Accessed 12 Dec 2016.

73. The American Gut Project via Qiita. https://qiita.ucsd.edu/study/description/ 10317. Accessed 10 June 2017.

74. Dereeper A, Guignon V, Blanc G, Audic S, Buffet S, Chevenet F, Dufayard JF, Guindon S, Lefort V, Lescot M, et al. Phylogeny.fr: robust phylogenetic analysis for the non-specialist. Nucleic Acids Res. 2008;36:W465-9.

75. Dereeper A, Audic S, Claverie JM, Blanc G. BLAST-EXPLORER helps you building datasets for phylogenetic analysis. BMC Evol Biol. 2010;10:8.

76. Edgar RC. MUSCLE: multiple sequence alignment with high accuracy and high throughput. Nucleic Acids Res. 2004;32:1792-7.

77. Castresana J. Selection of conserved blocks from multiple alignments for their use in phylogenetic analysis. Mol Biol Evol. 2000;17:540-52.

78. Guindon S, Gascuel O. A simple, fast, and accurate algorithm to estimate large phylogenies by maximum likelihood. Syst Biol. 2003;52:696-704.

79. Anisimova M, Gascuel O. Approximate likelihood-ratio test for branches: a fast, accurate, and powerful alternative. Syst Biol. 2006;55:539-52.

80. Chevenet F, Brun C, Banuls AL, Jacq B, Christen R. TreeDyn: towards dynamic graphics and annotations for analyses of trees. BMC Bioinformatics. 2006;7:439.

81. Phylogeny.fr documentation. http://www.phylogeny.fr/documentation.cgi oneclick. Accessed 17 May 2017.

82. Fitch WM. An improved method of testing for evolutionary homology. J Mol Biol. 1966;16:9-16

83. Gelman A, Hill J. Data analysis using regression and multilevel/hierarchical models. New York; Cambridge university press; 2007.

84. Akaike H. A new look at the statistical model identification. IEEE Trans Autom Control. 1974;19:716-23.

85. Schwarz G. Estimating the dimension of a model. Ann Stat. 1978;6:461-4.

86. Aitchison J. A new approach to null correlations of proportions. Math Geol. 1981;13:175-89.

\section{Submit your next manuscript to BioMed Central and we will help you at every step:}

- We accept pre-submission inquiries

- Our selector tool helps you to find the most relevant journal

- We provide round the clock customer support

- Convenient online submission

- Thorough peer review

- Inclusion in PubMed and all major indexing services

- Maximum visibility for your research

Submit your manuscript at www.biomedcentral.com/submit 